\title{
Criar en colores, nuevas experiencias: una intervención de agua y color con madres jóvenes de etnia gitana en Servicios Sociales de Fuentidueña, Villa de Vallecas
}

\author{
Raising in colors, new experiences: a water and color intervention \\ with young gypsy mothers in the Social Services of Fuentidueña, \\ Villa de Vallecas
}

\author{
Adelaida LARRAÍN. Universidad Complutense de Madrid (España). \\ adelarra@ucm.es \\ Fátima CORTÉS. CMSc de Villa de Vallecas (España). cortesff@madrid.es \\ Noemí ÁVILA. Universidad Complutense de Madrid (España). \\ noemi.avila@art.um.es
}

Resumen: Criar en colores, nuevas experiencias es un proyecto de arte y salud llevado a cabo en el distrito Villa de Vallecas, Madrid. La intervención, perteneciente al Programa de salud sexual y reproductiva, se encuadra en los programas de promoción y prevención de la salud de los Centros Municipales de Salud Comunitaria del Ayuntamiento de Madrid, en conjunto con el Centro de Servicios Sociales de la Comunidad de Madrid. Se trata de un programa desarrollado semanalmente entre los meses de enero a abril de 2018, en el Centro de Servicios Sociales de Fuentidueña de Villa de Vallecas. La intervención fue llevada a cabo por una profesional del arte y la salud, profesionales de la medicina de Madrid Salud, y los educadores sociales de familia del centro de servicios sociales. El taller se desarrolló con un grupo de mujeres, madres jóvenes de población gitana, beneficiarias de la prestación de renta mínima de inserción, RMI. El grupo denominado Aprendiendo a ser madres, se había formado hace dos años en el centro. Uno de los principales objetivos planteados fue continuar en la línea de formación de este grupo, y para ello se presentó un programa de arte (acuarela y técnicas al agua), a través del cual las mujeres desarrollarían con un lenguaje plástico y artístico, experiencias de bienestar, disfrute, encuentro con sus hijos/as y descubrimiento de nuevos espacios de salud. 
Palabras clave: acuarela, bienestar, empoderamiento, gitanas, madres, mujeres

\begin{abstract}
Raising in colors, new experiences is an art and health project carried out in the Villa de Vallecas district, Madrid. The intervention is part of the Sexual and Reproductive Health Program and constitutes one of the health promotion and prevention programs implemented by Municipal Health Centers of the City of Madrid in conjunction with Madrid's Community Social Services Center. The intervention was developed on a weekly basis between January and April 2018 in the Social Services Center of Fuentidueña, Villa de Vallecas, and was carried out by an artist and educator, medical professionals from Madrid Salud and social family educators from the social services center. The workshop was oriented to a group of young gypsy mothers, called Learning to be Mothers, who are beneficiaries of the minimum insertion income (RMI). The group was formed two years ago at the center. One of the main objectives was to continue the capacitation of this group. With this purpose, an art program (Watercolor and water techniques) was presented, and through it women would develop, with plastic and artistic languages, experiences of well-being, enjoyment, connecting with their children and discovering new health spaces.
\end{abstract}

Keywords: watercolor, wellness, empowerment, gypsy, mothers, women

\title{
Introducción y objetivos
}

El proyecto Criar en colores, nuevas experiencias, se enmarca en los programas de promoción y prevención de la salud de los Centros Municipales de Salud Comunitaria (CMSc) de Madrid Salud del Ayuntamiento de Madrid, en conjunto con el Centro de Servicios Sociales. Los CMSc son centros municipales especializados en la promoción de la salud y en la prevención de las enfermedades y otros problemas de salud. ${ }^{1}$

El Ayuntamiento de Madrid Salud se ocupa de la prevención y la promoción de la salud en la ciudad de Madrid mediante una red de dieciséis CMSc en la ciudad. Uno de ellos es el CMSc de Villa de Vallecas. Según la Carta de Otawa redactada en 1986 en la OMS, se define la promoción de la salud como un proceso que proporciona a las poblaciones los medios necesarios para ejercer un mayor control sobre su propia salud y así poder mejorarla.

La intervención se llevó a cabo en el Centro de Servicios Sociales Fuentidueña en Villa de Vallecas con un grupo de madres jóvenes de población gitana en conjunto con el CMSc de Villa de Vallecas. El Centro de Servicios Sociales pertenece al

1. Madrid Salud http://madridsalud.es 
Distrito Villa de Vallecas, atiende a toda la población de: Casco Histórico, barrio Uva y Cañada Real sector 6. En el centro trabajan 9 educadores de área y junta: 4 educadores sociales de familia de la junta municipal, y 5 educadores sociales de la Comunidad de Madrid, 15 tutores y trabajadores sociales de RMI (orientación y reinserción laboral), 5 profesionales de CAI (centro de actividades infantiles). El CMSc ha trabajado en conjunto con el equipo de Servicios Sociales desde hace 3 años; ofreciendo talleres de educación para la salud. Se han abordado temas como el cuidado y alimentación infantil, taller de educación para la sexualidad, relaciones afectivo-sexuales, anticoncepción, ALAS: alimentación, actividad física y salud y taller de prevención de accidentes y primeros auxilios. Se han impartido alrededor de 7 talleres desde el año 2015, con un total de 14 sesiones de 1 hora y media cronológica, con un promedio de 8 usuarias por sesiones.

El grupo de mujeres con que se va a trabajar se denomina: Aprendiendo a ser madres, es un grupo ya consolidado, todas madres jóvenes de etnia gitana con hijos/ as pequeños no escolarizados, de 0-3 años, con un nivel básico de alfabetización $\mathrm{y}$ en el que a lo largo de estos años se aprecia un interés por aprender cosas diferentes y una alta participación dentro de los talleres. Desde hace dos años se dirigen semanalmente los miércoles a cargo de dos educadores sociales de familia. Las usuarias que participan en el taller son todas mujeres jóvenes receptoras de RMI: renta mínima de inserción (prestación económica, integrada por la suma de una prestación mensual básica y un complemento variable, en función de los miembros que forman parte de la unidad de convivencia, con objeto de satisfacer las necesidades básicas de la misma, cuando éstas no pueden obtenerse del trabajo, o de pensiones y prestaciones de protección social). ${ }^{2}$

Para recibirla deben cumplir con la asistencia obligatoria a los talleres semanalmente. Muchas de ellas traen un justificante por la falta de asistencia por motivos la mayoría de las veces médicos.

Según el análisis de propuestas para la actuación de salud y comunidad gitana de la Dirección General de Salud Pública del Ministerio de Sanidad y Consumo y Fundación Secretariado Gitano: una de las características en la salud de las mujeres gitanas es que se destaca por su exceso de responsabilidades dentro y fuera del hogar, con la consiguiente aparición de síntomas de depresión, angustia y ansiedad en algunos casos. (Salud y comunidad gitana, 2006, p.16).

La mujer gitana se convierte fundamentalmente en cuidadora de los demás, dejando de lado sus propios cuidados. Este grupo se dedica básicamente al cuidado del

2. Comunidad de Madrid: convocatorias y prestaciones. http://www.madrid.org/cs/Satellite?c=CM_ConvocaPrestac_FA\&c $\mathrm{id}=1109168955327 \&$ definicion=Prestaciones + Sociales\&idListConsj=1109265444710\&pagename=ComunidadMadrid $\% 2 \mathrm{~F}$ Estructura\&tipoServicio=CM_ConvocaPrestac_FA. 
hogar y la familia y eso les ocupa todo su tiempo. En relación con el rol de género y las funciones de soporte familiar, la mujer gitana antepone sus obligaciones de cuidado, así como de sustento económico y familiar, a la demanda de atención clínica. (Ramos-Morcillo et al., 2015, p.5).

Uno de estos problemas fue los que se percibió cuando se escogió este grupo para trabajar con la intervención artística. Esta cuestión fue planteada por la médica del CMSc Fátima Cortés, quien había participado en varias intervenciones anteriores con las mujeres, pero nunca abordando el tema salud desde el enfoque artístico. Según la profesional, estas mujeres pueden y quieren seguir aprendiendo para mejorar su nivel de salud y bienestar, y el de sus familias, pero podría ser interesante hacerlo mediante una metodología diferente; y el enfoque artístico ser una buena oportunidad.

Con estas premisas, se plantea la intervención con tres objetivos:

1. Promocionar y activar la experiencia de salud entendida como bienestar y empoderamiento a través de la herramienta artística de la acuarela y sus técnicas al agua.

2. Generar un espacio de intervención, aprendizaje y encuentro con la acuarela para el bienestar y el disfrute en las usuarias.

3. Evaluar el impacto percibido en la participación de los talleres, mediante las escalas de Bienestar Mental WEMWBS $\subset$, la medida paraguas de emociones positivas y negativas (Positive and Negative Wellbeing Umbrella - Generic (C) UCL Museums 2013) y los espacios de reflexión.

\section{Método}

A continuación, se explican las diferentes etapas de proceder y organizar la investigación para la realización del proyecto.

Participantes:

Participaron un total de 15 madres con una edad promedio de 26 años (usuaria mayor 42 años, usuaria menor 21 años) y un total de 11 niños/as entre 1 y 3 años. Se realizó un trabajo en equipo entre la médica del CMSc, los dos educadores sociales, un voluntario y la estudiante de doctorado de Bellas Artes, Departamento de Didáctica de la Expresión Plástica. 
Tabla 1

Perfil participantes de la intervención Criar en colores, nuevas experiencias

CMSc Villa de Vallecas

Criar en Colores, nuevas experiencias

\begin{tabular}{lc}
\hline Perfil & $\begin{array}{c}\text { Madres jóvenes de población gitana de } \\
\text { servicios sociales perceptoras de RMI }\end{array}$ \\
$\mathrm{N}^{o}$ participantes usuarias - hijos/as & $15-11$ \\
$\mathrm{~N}^{\mathrm{o}}$ participantes personal Madrid Salud & 1 \\
$\mathrm{~N}^{o}$ participantes educadores sociales de familia & 2 \\
Voluntarios & 2 \\
Edad promedio usuarias - hijos/as & 26 años- 18 meses \\
Temporalización de la intervención & 2 meses - 8 sesiones \\
\hline
\end{tabular}

Temporalización

A continuación, se enuncian las principales fechas desde las primeras reuniones previas a comenzar con la intervención.

- 30 de noviembre: presentación a los profesionales del CMSc anteriores programas de arte y salud como Criar en colores de madres jóvenes españolas y latinoamericanas con sus bebés. Se planifica la posibilidad de continuar la intervención con otro colectivo como población gitana.

- 18 de diciembre: segunda reunión con el equipo, se presenta el nuevo proyecto Criar en colores, nuevas experiencias a los profesionales del CMSc y a los educadores de familia del Centro de Servicios Sociales de Fuentidueña.

- 10 enero: ejecución y pintura de la diana de los paraguas de las emociones.

- Primeras cuatro sesiones: 31 enero, 7 febrero, 14 febrero, 21 febrero.

- 28 febrero: reunión de evaluación de las primeras cuatro sesiones con los educadores sociales y la médica del CMSc.

- 18 abril: preparación de montaje para exposición colectiva en el Centro Juvenil El Sitio de mi Recreo.

- 7 marzo: evaluación final de curso.

- 9 mayo: montaje y muestra final Centro Juvenil El Sitio de mi Recreo.

- 16 mayo: entrevista a los educadores sobre la experiencia en el proyecto Criar en colores, nuevas experiencias

- 5 junio: desmontaje. 
- 15 junio: visita guiada al Museo Thyssen-Bornemisza con el educador. Se gestionó la financiación de la visita al museo y el paseo final de curso con el Servicio de Comunicación de Madrid Salud.

\section{Herramientas de recogida de datos}

Además de las propias actividades realizadas durante las sesiones con las participantes respecto al uso de la acuarela y técnicas al agua; también han sido necesarias otras herramientas y actividades.

\section{Herramientas de recogida de datos}

- Entrevista cualitativa a los profesionales

- Observación participante durante las sesiones

- Registro en cuaderno de campo

- Registro fotográfico de los procesos y resultados artísticos

- Reuniones previas y posteriores con el equipo de trabajo

- Tarjetas de bienestar en dinámicas grupales

- UCL Museums Wellbeing Measures Toolkit (C)

- Warwick-Edinburgh Mental Well-being Scale (C)

Entrevista semiestructurada a los/las profesionales. Se realiza una entrevista semiestructurada a los/las profesionales de Servicio Sociales de Fuentidueña; los educadores sociales de familia. En ella se abordan temas sobre la relación de trabajo entre Madrid Salud y Servicios Sociales, los proyectos y trabajos abordados, la población diana, y el grupo Aprendiendo a ser madres; que se lleva desarrollando desde el 2012. También se realiza una entrevista a la médica del CMSc. La profesional lleva trabajando desde el año 2012 con población gitana del sector de Villa de Vallecas y Cañada. En la entrevista se describe como se ha desarrollado el proyecto de arte y salud con el grupo de usuarias de población gitana y sus resultados positivos.

Observación participante durante las sesiones y registro en cuaderno de campo. La observación y el registro en el diario de campo es una herramienta muy valiosa ya que en ella se analizan detalles, comportamientos, que luego permiten visualizar claramente los resultados.

Registro fotográfico de los procesos y resultados artísticos. El registro fotográfico es una herramienta visual que permite ver el proceso y resultado plástico en acuarela y técnicas al agua. Con previa autorización del consentimiento firmado se pudieron sacar fotos para explorar momentos de las usuarias en las intervenciones, durante la exposición y también de los trabajos plásticos en acuarela. 
Reuniones previas y posteriores con el equipo de trabajo. Se realizaron reuniones con el equipo de profesionales con el que se trabajó antes de comenzar el proyecto para ir afinando los objetivos, periodicidad del programa, contenidos, materiales, escalas y métodos de evaluación.

\section{Tarjetas de bienestar en dinámicas grupales}

Al término de algunas sesiones, se realizó una dinámica grupal de conversación haciendo uso de las tarjetas de bienestar: afecto, optimismo, cariño, disfrute, etc... De tonos rojos, rosas, violetas, colores que respondían a los gustos de las usuarias. Algunas palabras como (apego, cariño, ocio, vínculo social) fueron sustituidas por (afecto, amor, diversión y relación social), para obtener una mayor comprensión y cercanía a su lenguaje. Las tarjetas permitieron generar una participación hacia la comunicación más activa y profunda sobre la experiencia del taller. De esta manera fluyó una mayor dinámica de interacción donde las usuarias se sintieron seguras al expresar sus opiniones. Esta actividad, les generó autoconfianza y bienestar, quedando demostrado en el pequeño testimonio de cada participante, manifestando el porqué habían elegido cada palabra.

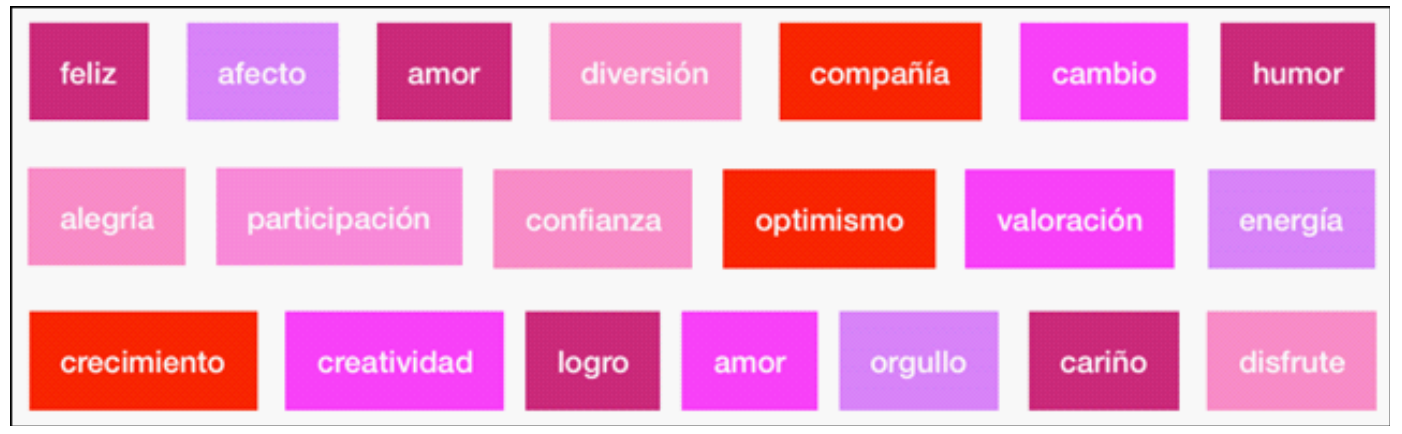

Figura 1. Tarjetas de bienestar

UCL Museum Wellbeing Measures Toolkit: Positive and Negative Wellbeing Umbrella - Generic (C UCL Museums 2013, es una investigación realizada por la doctora Linda Thomson y Helen Chatterjee de La University College de Londres. Consiste en un conjunto de 4 escalas de medición utilizadas para evaluar los niveles de bienestar derivados de la participación en actividades de museos y galerias que se han probado en todo el Reino Unido. (Linda J. Thomson - Helen J. Chatterjee). Con previa autorización de Linda Thomson se realizaron las traducciones de los términos del ingles al español y luego se aplicaron con las usuarias en forma interactiva como juego de diana. La diana consistía en una adaptación de las medidas genérica positiva y negativa, agrandada al 200\% y pintadas en acuarela según los colores de los originales. Las usuarias fueron colocando la puntuación de sus emociones según los números indicados del 1 al 5 directamente sobre la diana. Figuraban las emociones positivas: activa, alerta, entusiasmada, emocionada, feliz, inspirada; y las emociones negativas: afligida, asustada, infeliz, irritable, enfadada, nerviosa. Los términos que fueron dificultosos para comprender se explicaron 
mediante ejemplos: entusiasmado (que tienes ganas de continuar, contenta con la tarea), inspirada (creativa), activa (con energía), feliz (contenta, he disfrutado), alerta (atenta). La evaluación fue difícil llevar a cabo, ya que en general son poco disciplinadas, su nivel de dispersión es alto, con escasa concentración al final de las sesiones; sin embargo, era importante dejar registrada la evaluación semanalmente, ya que la continuidad le da el valor a la intervención.

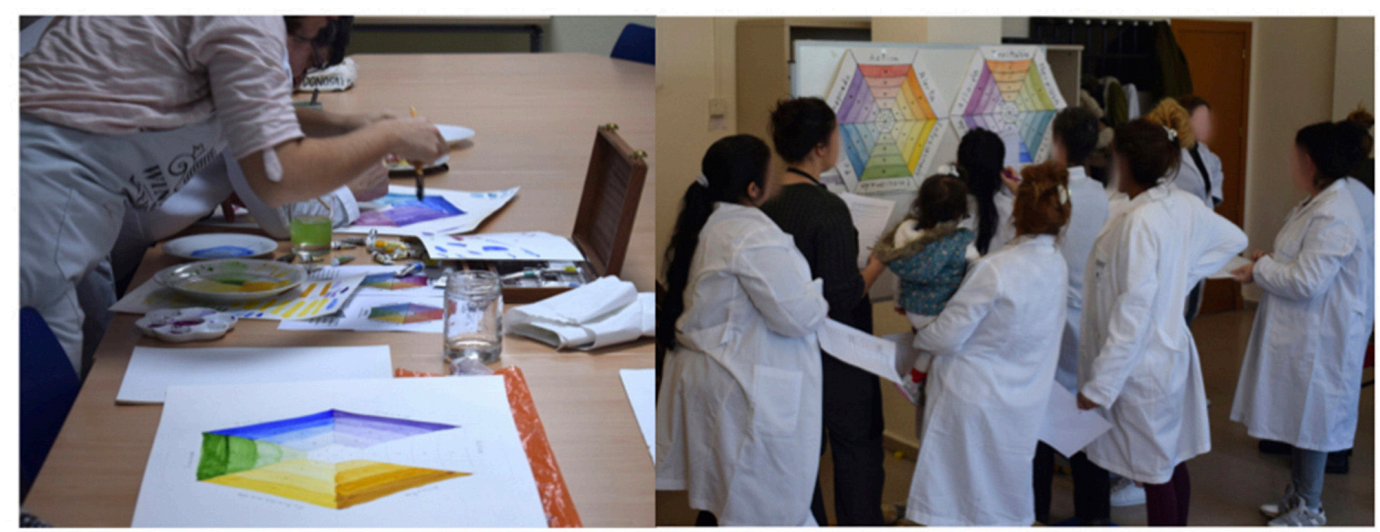

Figura 2. Pintura de "paraguas de las emociones" - usuarias durante la evaluación y la participación en la diana.
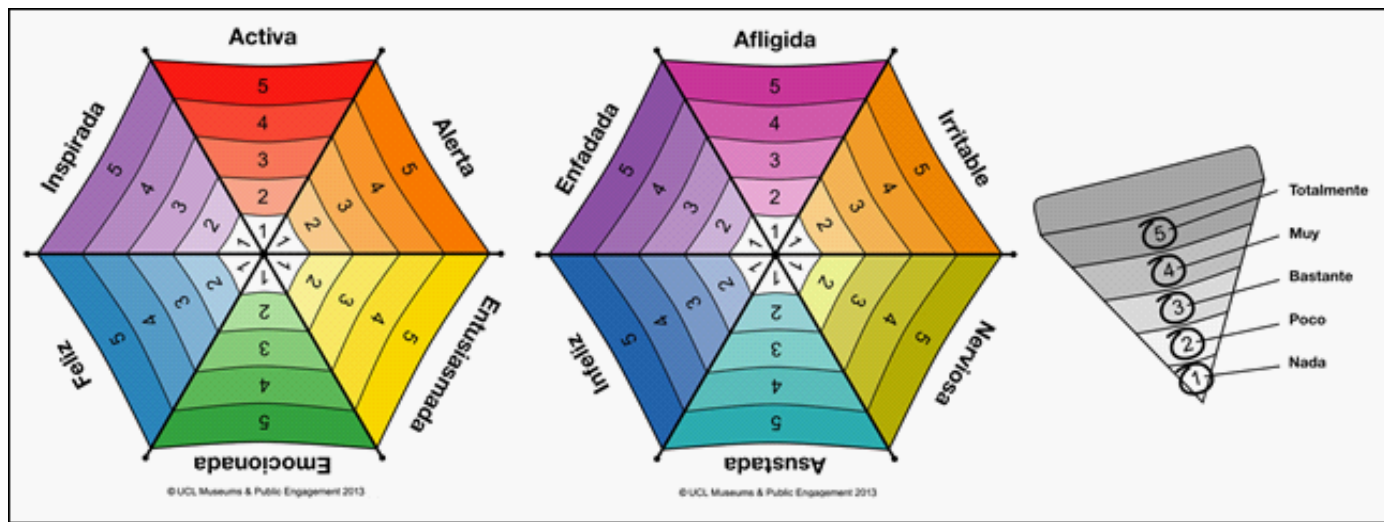

Figura 3. Positive and Negative Wellbeing Umbrella - Generic@ UCL Museums

\section{Warwick-Edinburgh Mental Well-being Scale $\subset$}

La escala de bienestar mental de Warwick-Edimburgo, fue desarrollada para permitir el monitoreo del bienestar en la población general y la evaluación de proyectos, programas y políticas que apuntan a su mejora. WEMWBS(C es una escala de 14 items con 5 categorías de respuestas, sumadas para proporcionar una puntuación única que va desde 14 a 70. Los ítems están redactados de manera positiva y cubren los aspectos de bienestar mental que funcionan y sienten. El WEMWBS $\subset$ ha sido traducido a varios idiomas y algunos de ellos han sido validados psicométrica y cualitativamente. ${ }^{3}$ Para descargar la versión española se solicitó con previa

3. Para más información: https://warwick.ac.uk/fac/med/research/platform/wemwbs/ 
presentación del proyecto la autorización a BiblioPRO: Biblioteca Virtual de referencia científica internacional de los cuestionarios en español de Resultados Percibidos por los Pacientes. (PRO - Patient Reported Oucomes). ${ }^{4}$

El cuestionario WEMWBS, se entregó al finalizar la primera y última sesión, resultando algunas preguntas y conceptos difíciles de comprender y leer por parte de las usuarias. Algunos enunciados fueron reemplazados a un lenguaje más coloquial y así leídos en forma oral ante el grupo de participantes. Por ejemplo: ¿Me he sentido útil? por ¿Soy de ayuda para los demás? - ¿Me he sentido optimista respecto al futuro? por ¿Me siento feliz cuando pienso en mañana.

Alas usuarias les cuesta manifestar espontáneamente su opinión y la mayoría responde inicialmente "no sé" buscando con la mirada la aprobación de sus educadores. Todo lo que sea la opinión propia, no se atreven ni se permiten expresarlo, menos aún escribirlo. La cultura gitana transmite la enseñanza en forma oral de generación en generación y además el nivel educativo de las participantes es bajo. De ahí la dificultad para la comprensión lectora de los enunciados de WEMWBSC

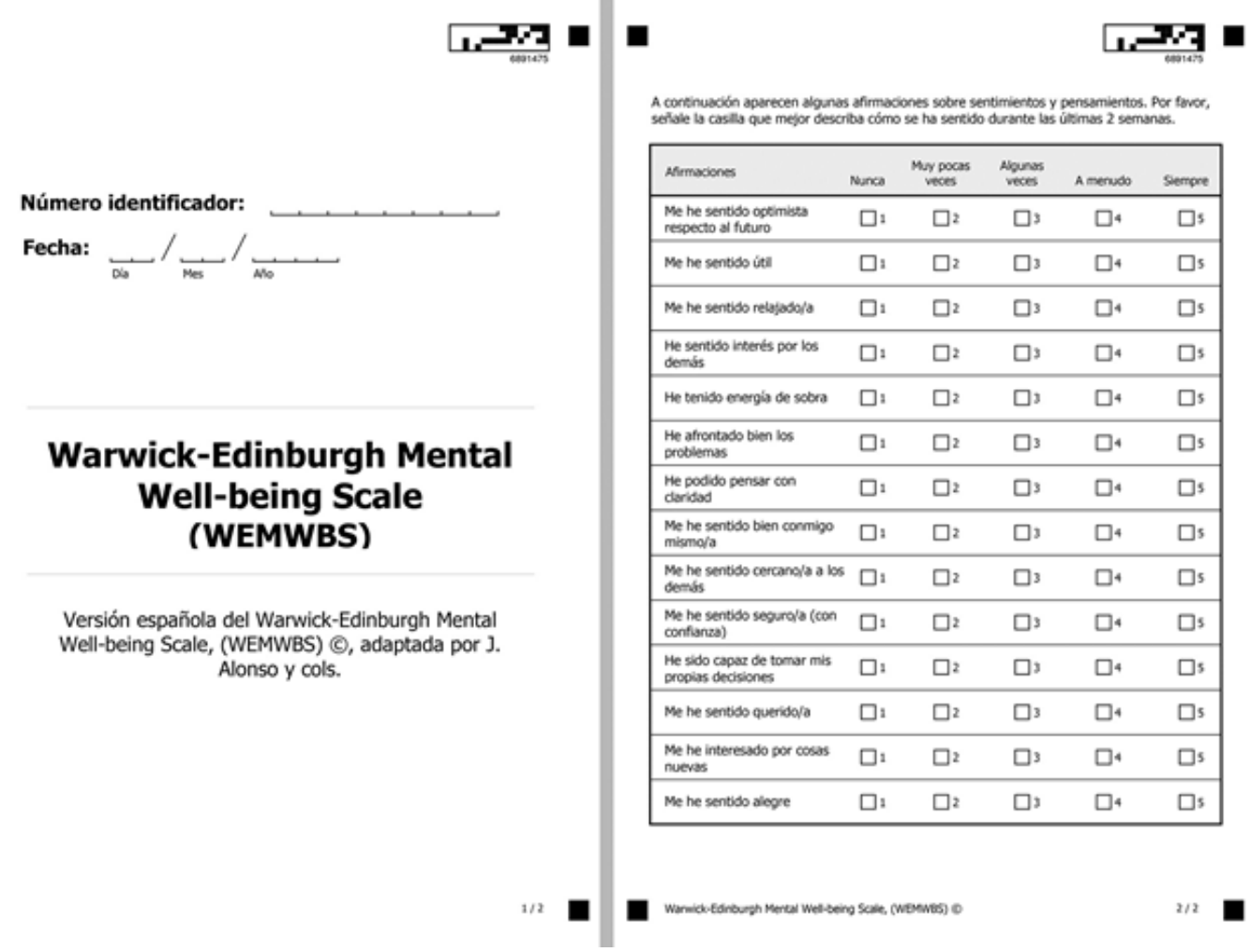

Figura 4. Versión española de Warwick-Edinburgh Mental Well-being Scale, (WEMWBS) C), adaptada por J. Alonso y cols.

4. Para más información: https://www.bibliopro.org 


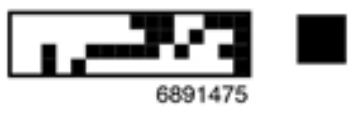

A continuación aparecen algunas afirmaciones sobre sentimientos y pensamientos. Por favor, señale la casilla que mejor describa cómo se ha sentido durante las últimas 2 semanas.

\begin{tabular}{|c|c|c|c|c|c|}
\hline Afirmaciones & Nunca & $\begin{array}{l}\text { Muy pocas } \\
\text { veces }\end{array}$ & $\begin{array}{l}\text { Algunas } \\
\text { veces }\end{array}$ & A menudo & Siempre \\
\hline $\begin{array}{l}\text { Me he sentido optimista } \\
\text { respecto al futuro }\end{array}$ & $\square 1$ & $\square 2$ & $\square 3$ & $\square 4$ & $\square 5$ \\
\hline Me he sentido útil & $\square 1$ & $\square_{2}$ & $\square_{3}$ & $\square_{4}$ & $\square 5$ \\
\hline Me he sentido relajado/a & $\square 1$ & $\square 2$ & $\square 3$ & $\square_{4}$ & $\square 5$ \\
\hline $\begin{array}{l}\text { He sentido interés por los } \\
\text { demás }\end{array}$ & $\square 1$ & $\square_{2}$ & $\square_{3}$ & $\square_{4}$ & $\square 5$ \\
\hline He tenido energía de sobra & $\square 1$ & $\square^{2}$ & $\square_{3}$ & $\square_{4}$ & $\square 5$ \\
\hline $\begin{array}{l}\text { He afrontado bien los } \\
\text { problemas }\end{array}$ & $\square 1$ & $\square 2$ & $\square 3$ & $\square_{4}$ & $\square 5$ \\
\hline $\begin{array}{l}\text { He podido pensar con } \\
\text { claridad }\end{array}$ & $\square 1$ & $\square 2$ & $\square 3$ & $\square 4$ & $\square 5$ \\
\hline $\begin{array}{l}\text { Me he sentido bien conmigo } \\
\text { mismo/a }\end{array}$ & $\square 1$ & $\square 2$ & $\square 3$ & $\square 4$ & $\square 5$ \\
\hline $\begin{array}{l}\text { Me he sentido cercano/a a los } \\
\text { demás }\end{array}$ & $\square 1$ & $\square 2$ & $\square_{3}$ & $\square 4$ & $\square 5$ \\
\hline $\begin{array}{l}\text { Me he sentido seguro/a (con } \\
\text { confianza) }\end{array}$ & $\square 1$ & $\square 2$ & $\square 3$ & $\square 4$ & $\square 5$ \\
\hline $\begin{array}{l}\text { He sido capaz de tomar mis } \\
\text { propias decisiones }\end{array}$ & $\square 1$ & $\square 2$ & $\square_{3}$ & $\square_{4}$ & $\square 5$ \\
\hline Me he sentido querido/a & $\square 1$ & $\square_{2}$ & $\square_{3}$ & $\square_{4}$ & $\square 5$ \\
\hline $\begin{array}{l}\text { Me he interesado por cosas } \\
\text { nuevas }\end{array}$ & $\square 1$ & $\square_{2}$ & $\square 3$ & $\square 4$ & $\square 5$ \\
\hline Me he sentido alegre & $\square 1$ & $\square 2$ & $\square_{3}$ & $\square_{4}$ & $\square 5$ \\
\hline
\end{tabular}




\section{Diseño del programa de intervención}

Teniendo en cuenta todo el diseño metodológico anteriormente descrito, se diseñó el programa Criar en colores, nuevas experiencias, con los siguientes objetivos y contenidos.

\section{Objetivos del programa}

- Disfrutar experimentando y practicando con técnicas artísticas al agua principalmente la acuarela, material artístico desconocido para las algunas usuarias.

- Desarrollar habilidades y destrezas artísticas mediante la acuarela para mejorar su autoestima, confianza e independencia en su vida diaria.

- Compartir una experiencia enriquecedora a través de la participación con sus hijos/as en los talleres de arte y salud.

- Descubrir un nuevo espacio de encuentro materno - filial.

- Valorar la creación personal y de sus compañeras con una actitud crítica y respetuosa.

- Fortalecer relaciones sociales y vínculos con sus pares.

- Participar en una exposición comunitaria en el Centro Joven El Sitio de mi Recreo; para dar a conocer los trabajos realizados durante el curso.

- Asistir y participar en la salida y paseo final de curso: visita guiada al Museo Nacional Thyssen-Bornemisza.

\section{Breve descripción de las sesiones y sus contenidos}

$1^{\mathrm{a}}$ sesión Descubriendo el pincel: pinceladas y líneas

La primera sesión consistió en un acercamiento y breve conocimiento sobre los tipos de pinceles. Cómo usarlos con acuarela y las posibilidades de pinceladas y líneas sobre papel húmedo. Se usó formato mayor al corriente para que pudieran ejercitar con más libertad.

Cuando vean mis hijas el dibujo, van a ser que les compre acuarela.

(cita de usuaria durante la sesión)

$2^{\text {a }}$ sesión Color transparencia, color goteo, color soplo, color salpicadura, color chorreo

Se trabaja en el suelo sobre las colchonetas, con los materiales organizados y dispuestos para la actividad. Previo a comenzar el ejercicio, se realiza un ejemplo para que visualicen de manera clara las técnicas y modos de uso. Se escoge un color para usar sobre el papel humedecido con las diferentes técnicas como transparencia, goteo, soplo, salpicado y chorreo. 
$3^{\mathrm{a}}$ sesión Mezclas de color: color primario y secundario

Las usuarias aprendieron a distinguir los colores primarios y secundarios, nombrando ejemplos de la naturaleza. Luego se practicó la mezcla entre ellos para obtener nuevos resultados.

$4^{\mathrm{a}}$ sesión Pintura de frutas y verduras

Cada usuaria trajo de su hogar una fruta o verdura a su elección. Se utilizaron como modelo para observarlas y pintar sus formas y colores sobre el papel humedecido, con técnicas al agua como acuarela, tinta, lápices acuarelables, cera al agua.

$5^{\mathrm{a}}$ sesión Graduación de colores, tonalidades

Se practicó el ejercicio de la graduación de tonos y colores desde los más oscuros a los claros y viceversa.

$6^{\mathrm{a}}$ sesión Composición de calabazas y estampación de hojas secas.

La actividad consistió en realizar una composición escogiendo una calabaza como modelo para pintar y hojas secas de árbol para estampar con color sobre el papel humedecido. Se enseñó la técnica de estampación, produciendo expectación en las usuarias.

$7^{\mathrm{a}}$ sesión Flores

Para la actividad se les había solicitado la semana anterior una flor natural para pintar. Se ubican las flores al centro para observarlas y pintarlas con las técnicas al agua.

$8^{\mathrm{a}}$ sesión Preparación y montaje de la muestra colectiva en el Centro Juvenil.

\section{Resultados}

Partiendo del objetivo principal que articula la intervención: la mejora del bienestar en el grupo de mujeres, y a partir de los datos observados y obtenidos; los resultados se han articulado en los seis ítems:

- Asistencia / puntualidad: este ítem permite reflejar el interés y compromiso con el programa.

- Concentración y atención, durante las actividades y sesiones.

- Disfrute de las actividades del programa.

- Empoderamiento y reconocimiento de sus capacidades en el ámbito público.

- Integración y relación con los hijos/as.

- Mejora del bienestar 


\section{Asistencia / Puntualidad}

Las usuarias por lo general no se caracterizaron por ser puntuales, iban llegando a la sesión en forma desordenada e impuntual, lo que dificulta la tarea para comenzar todas a la vez. A pesar de esto, y en relación con el curso y experiencias anteriores en otros talleres impartidos por el CMSc, esta intervención se valoró positivamente como la que tuvo más duración en el tiempo con un porcentaje importante de asistencia.

Más de la mitad de las madres tuvo una participación en el 50\% de las sesiones o mayor. Sin embargo, hubo un pequeño porcentaje que por distintas circunstancias tuvieron dificultades para acudir de forma continua; la mayoría por razones médicas (con entrega posterior de justificante a los educadores). La asistencia a cada taller nunca fue del grupo completo $y$, por tanto, tampoco fueron las mismas usuarias las que acudían a cada una de las sesiones.

Al ser receptoras de RMI (Renta Mínima de Inserción), deben cumplir asistencia obligatoria a los talleres que se imparten en los Servicios Sociales. La amenaza de que pudieran dejar de recibir la ayuda de la RMI, si existen constantes inasistencias, es un problema, ya que el taller se convierte en un medio obligatorio y no un deseo voluntario de ir para aprender. Se ha observado que cuando justifican por una razón médica se les coloca una anotación.

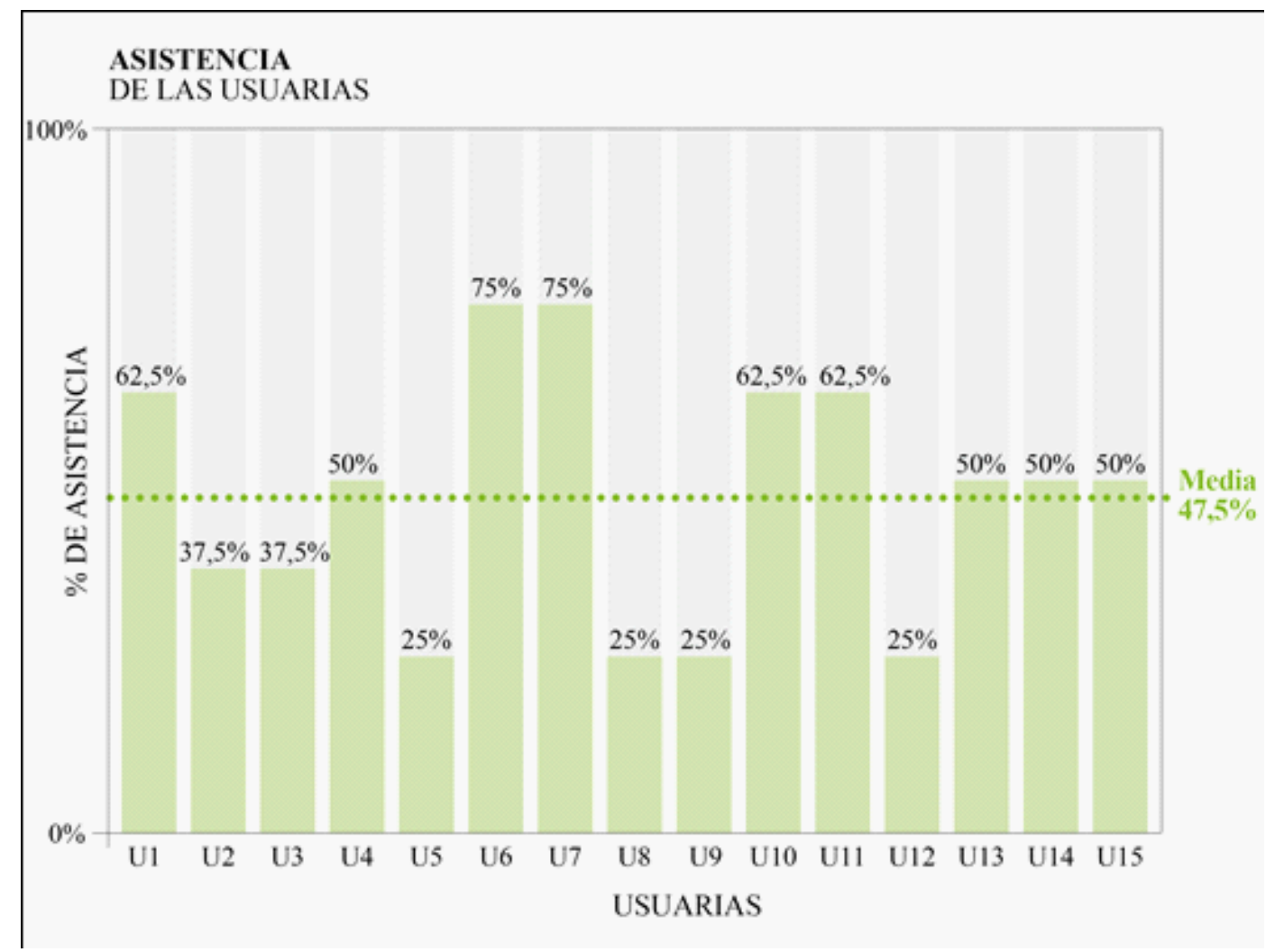

Figura 5. Gráfico de asistencia de las usuarias 
LARRAÍN, CORTÉS \& ÁVILA / Criar en colores, nuevas experiencias: una intervención de agua y color con madres jóvenes de etnia gitana en Servicios Sociales de Fuentidueña, Villa de Vallecas

\section{Concentración y atención durante las actividades y sesiones}

El taller se desarrolló al interior de una sala espaciosa, iluminada y bien abastecida de mobiliario (mesas, sillas, pizarra, colchonetas). Antes de dar comienzo a la actividad, se disponía un tiempo para la organización del espacio y los materiales. Generalmente, las sesiones se desarrollaron ubicándose las participantes en el suelo sobre colchonetas, con los materiales al centro. De este modo, se facilitó la participación de los niños en la actividad y la interacción de las madres con sus hijos/as en el ejercicio de agua y color. La integración de la acuarela en los talleres para promoción de la salud madre-hijo/a ha condicionado una forma novedosa de trabajo en este contexto, ya que era la primera vez que se trabajaba pintando sobre colchonetas sobre el suelo. Esta modalidad dio cabida a que la mayoría de las sesiones se desarrollaran en un ambiente distendido y relajado, incluyendo a veces en la pintura a los niños/as de una manera tranquila y lúdica, obteniendo una respuesta emocional positiva por parte de ellos/as.

Se observó dificultad para prestar atención a las instrucciones y los momentos de concentración fueron cortos. Sin embargo, las sesiones se desarrollaron con total fluidez, ya que comprendieron las instrucciones básicas del ejercicio acompañado de un lenguaje sencillo de comunicación. Lo más recomendable era comenzar por tareas simples y luego cada una según sus habilidades creativas continuaba la expresión.

La actividad se inicia con ejemplos concretos, que facilitan la comprensión del ejercicio, y luego cada usuaria continúa con la creación propia. Las usuarias son muy temerosas para tomar iniciativas y esperan instrucciones didácticas. Aunque su capacidad de concentración no se compara al de otros grupos de intervención de arte se considera que las participantes mantienen un tiempo mínimo en el que conectan con la tarea y centran su atención en el ejercicio. Los educadores de servicios sociales han comentado que mantienen más silencio y concentración aquí que en otras actividades realizadas en el Centro. El tiempo cronológico de trabajo y la dinámica de grupo está determinada por la concentración y comportamiento de los niños/as y los horarios de quehaceres domésticos y no duran, en general, más de una una hora. Antes de finalizar la sesión, se dejan 5 a 7 minutos para realizar la escala de las emociones. Esta fue difícil realizar al finalizar la actividad, las usuarias estaban desconcentradas, poco disciplinadas y con prisa por marchar. 


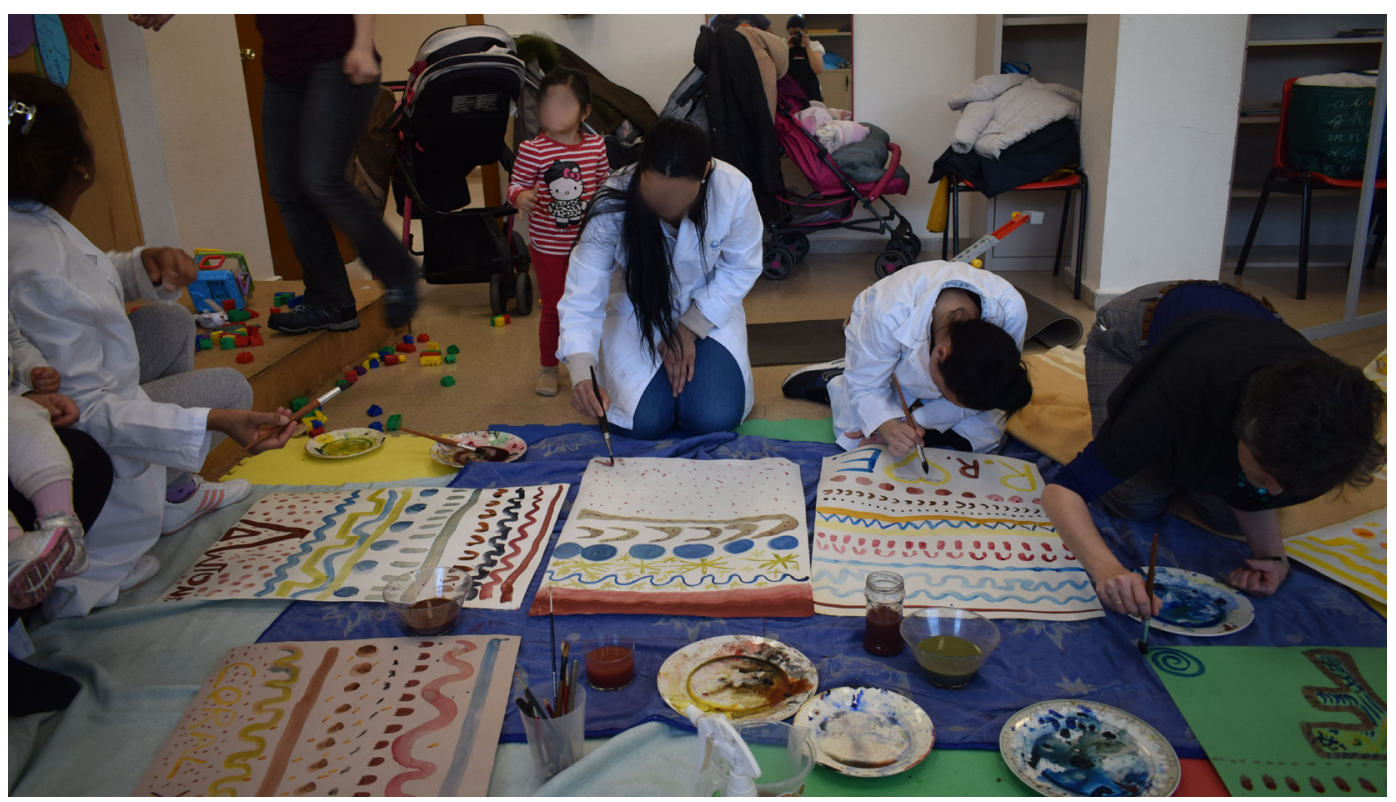

Figura 6. $1^{\mathrm{a}}$ sesión: descubrimiento del pincel y el color con la acuarela.

Disfrute de las actividades del programa. La intervención que se programó para las madres vino a compensar las inquietudes y el tiempo que no encuentran en el hogar y así reservar espacios personales de actividad para ellas. El taller supuso un tiempo de desconexión y disfrute con el agua y el color. La ayuda de los voluntarios con los niños/as fue muy importante ya que permitía que las mujeres estuvieran más conectadas con la tarea y el proceso creativo. Las usuarias reclaman un momento de evasión, autocuidado y distracción de los quehaceres de la vida cotidiana, ya que dicen no tener tiempo para sí mismas. Están todo el día dedicadas al cuidado de sus hijos/as, pero con poco disfrute del juego con ellos. Hay ejemplos de cuando se hacen excursiones, manifestar de querer ir sin los hijos/as para así descansar. En el espacio del taller expresan y esperan encontrar en la pintura un lugar de desconexión. La médica que acompañó la intervención señaló que era importante ofrecerles una actividad educativa a la vez de lúdica, que las empoderara y les rebelara un autoconocimiento sobre otras habilidades desconocidas. Una actividad que les brindara capacidad para disfrutar del arte y la belleza y les abriera nuevas posibilidades dentro de sus vidas, así mismo también podría servir para darles un protagonismo delante de sus familias.

La herramienta utilizada para medir las emociones positivas y negativas Positive and Negative Wellbeing Umbrella-Generic (C) UCL Museums 2013; tiene potencialmente múltiples usos y dimensiones. Las medidas pueden ser tomadas en diferentes intervalos de tiempo, por ejemplo, en el inicio de un programa de actividades, después de varias semanas y al final, etc. (Thomson, Chatterjee, 2015, p.52).

En la presente investigación, se ha utilizado la dimensión para visualizar los cambios en cuanto a las emociones positivas y negativas individuales de cada participante, tomando los datos de cada una de ellas en la primera y en la última sesión. Los 
LARRAÍN, CORTÉS \& ÁVILA / Criar en colores, nuevas experiencias: una intervención de agua y color con madres jóvenes de etnia gitana en Servicios Sociales de Fuentidueña, Villa de Vallecas

gráficos reflejan que la mayoría de las usuarias aumentan sus emociones positivas entre la primera y última sesión y, en el mismo período, disminuyen las negativas.

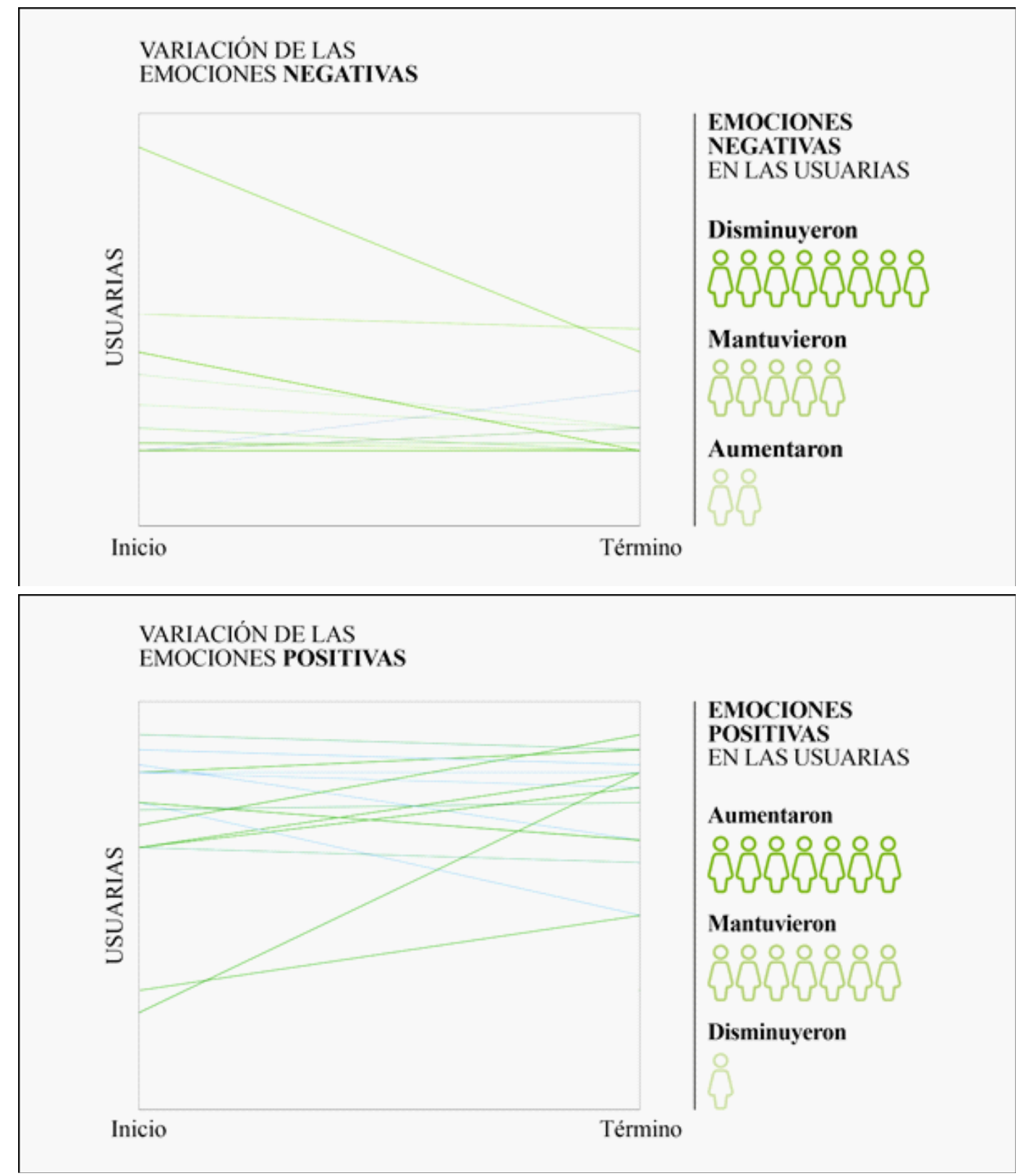

Figuras 7 y 8 . Resultados de las emociones positivas y negativas de cada una de las participantes, tomando los datos en la primera y en la última sesión

Empoderamiento y reconocimiento de sus capacidades en el ámbito público. Para desarrollar la identidad, las personas buscan el reconocimiento en el grupo al que pertenecen. En la población gitana la familia es la institución principal de la que parte este reconocimiento. Las pautas sociales de este grupo de población hacen que la autoridad y el reconocimiento siempre se le asigne al hombre estando la mujer subordinada al mismo. La mujer adquiere estatus social y respeto mediante el matrimonio y la maternidad. El protagonismo femenino se da siempre en el ámbito 
de lo privado, no en el público y las funciones están relacionadas con el cuidado del hogar y la familia.

El taller se ha enfocado en brindarles herramientas que le permitan a la mujer sentirse empoderada mediante las actividades de arte que se realizan semanalmente para mejorar su autoestima y generar espacios de bienestar personal y grupal.

La exposición final colectiva con las obras de todas las usuarias que se ha exhibido en el Centro Juvenil El Sitio de mi Recreo les ha brindado una oportunidad de reconocimiento y mérito frente al público. La exposición, significó una ocasión e instancia para conocer el espacio cultural que ofrece actividades con acceso gratuito hasta los 30 años. Los cursos que se imparten en el Centro Juvenil tienen corta duración, lo que supone un beneficio y facilidad para asistir. Se les animó y propuso que invitaran a sus maridos y familia a la muestra final para que conocieran el trabajo del curso, se involucraran y de una manera indirecta participaran en la actividad de sus mujeres. La médica animaba y decía con estas palabras Nos gustaría plantearles que los primeros que vieran la muestra de pintura fueran sus maridos y familiares.

En una de las sesiones se leyó una reseña de Lita Cabellut, artista multidisciplinar gitana española que trabaja con el óleo sobre lienzo, dibujos en papel, escultura, para que conocieran y tuvieran una referencia, de que una de las pintoras más importantes a nivel internacional, es gitana. Cabellut (2016) expresa:

He dado muchas vueltas y me he esforzado mucho con el hincapié de ser mujer y gitana en un oficio como la pintura, donde sólo destacan hombres. Ahora me están empezando a llamar para que venga aquí a exponer mis cuadros", afirma la pintora. Pienso que es porque estamos en un momento en el que necesitamos ejemplos de personas que han salido adelante después de estar en el pozo más hondo, personas que vendan ilusión, y si mi arte puede inspirar y dar aliento, yo seré muy feliz. ${ }^{5}$

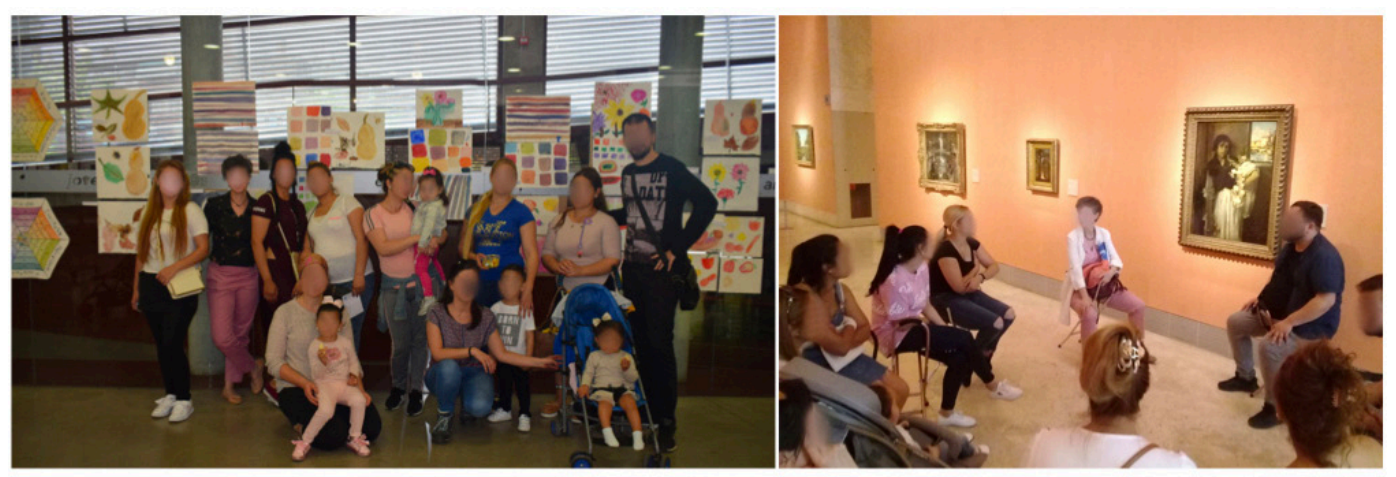

Figura 9. Grupo Aprendiendo a ser madres, en la muestra final - visita al Museo Nacional ThyssenBornemisza

5. De La Cal, L. (2016). Lita la pintora española más cotizada es gitana, El Mundo, Recuperado de http://www.elmundo.es/ cronica/2016/04/16/5707e8e3268e3e06298b4677.html 
LARRAÍN, CORTÉS \& ÁVILA / Criar en colores, nuevas experiencias: una intervención de agua y color con madres jóvenes de etnia gitana en Servicios Sociales de Fuentidueña, Villa de Vallecas

\section{Integración y relación con los hijos/as}

Se les ofrece en el taller una nueva forma y espacio de integración con sus hijos/as, sin embargo, se da un fenómeno contradictorio. Por una parte, necesitan encontrar un espacio para desconectar de sus hijos/as y las tareas domésticas y por otro lado les es dificultoso no estar pendientes de ellos/as, ya que los niños/as en general están apegados a sus madres. La ayuda de los voluntarios fue importante ya que ellos se ocupaban de los niños/as durante el taller. Las mujeres respondieron a la intervención como un momento de descanso y desconexión, ya que ellas constantemente asumen el rol del cuidado de sus hijos/as. En la ronda de palabras con las tarjetas de bienestar una usuaria escogió: descanso Te desconectas de los hijos, puedo hacer lo que quiero. Otra usuaria escogió calma Estoy muy estresada, aqui me relajo, necesitaría más este espacio, pero sola, es muy sano para mí. Arbex señala:

Dentro y fuera del hogar la mujer gitana es la principal cuidadora y responsable de sus hijos, adjudicándose toda la responsabilidad y el papel a ella. Ella ocupa un papel muy importante dentro de la familia. Se encarga de educar; criar y cuidar a los hijos y familiares, (padres, hermanos, tíos...), convirtiéndose en un agente de transmisión de normas, valores y costumbres. (Arbex, 2000, p.7)

Dedican gran parte del día al cuidado de sus hijos, pero con poco disfrute del juego y ocio. Por otra parte, por determinantes culturales, le cuesta poner límites a sus hijos y eso dificulta la comunicación y la relación. La médica del CMSc le parecía que esta actividad compartida de agua y color podía favorecer una relación materno-filial diferente en la que era posible compartir en espacios de ocio, siempre respetando ciertas normas.

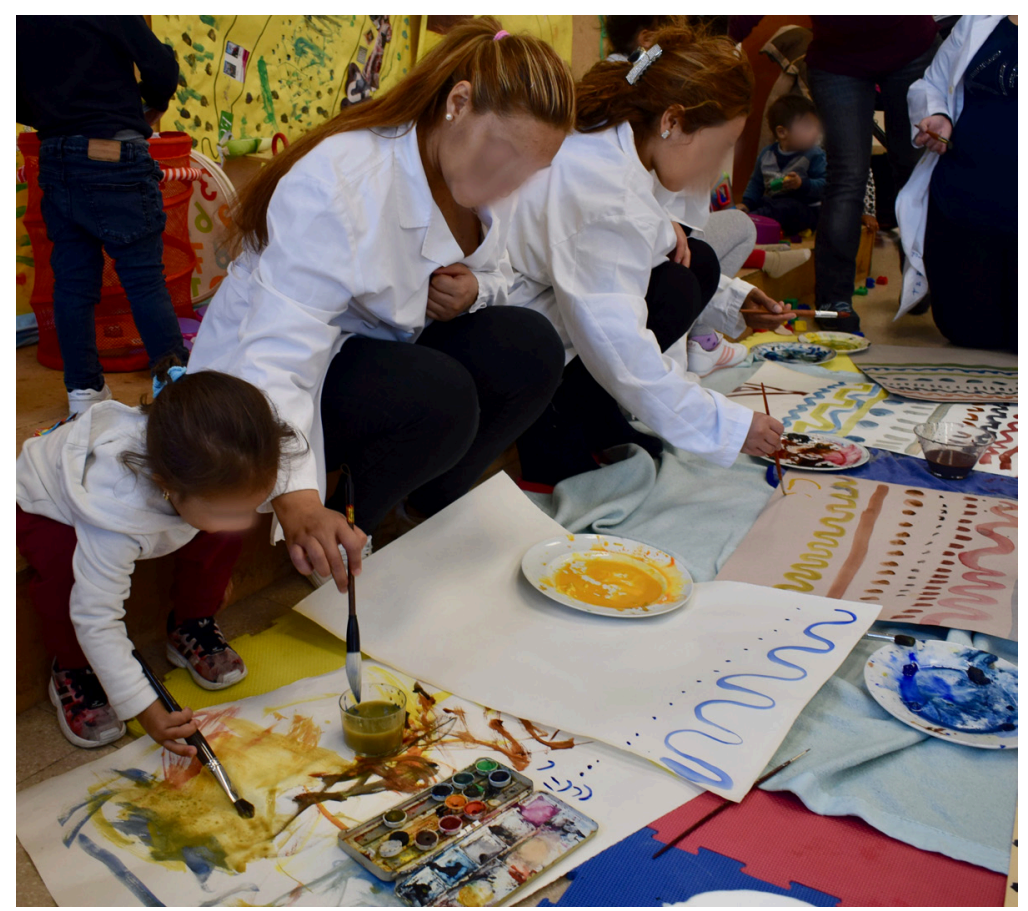

Figura 10. Usuarias disfrutando de la sesión del taller de arte con sus hijos/as 
Mejora del bienestar. Los resultados de WEMEBSC demostraron que casi la mayoría de las usuarias aumentó su estado de bienestar después de la intervención grupal semanal coincidiendo que varias de ellas han tenido una asistencia del más del $50 \%$ en el curso. Un 66,6\% de las usuarias aumentó su estado de bienestar desde los inicios del curso hasta el final de la intervención, un 26,6\% no demostró ese aumento y solo un $6,6 \%$ de las usuarias se mantuvo con el mismo nivel.

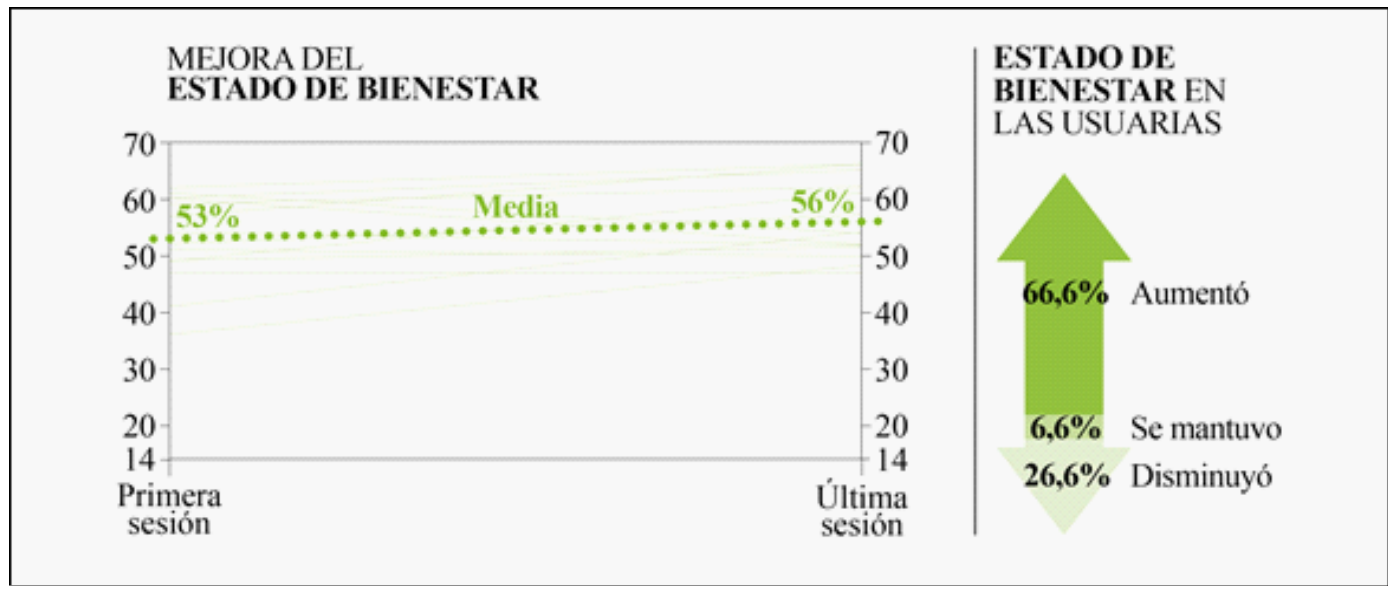

Figura 11. Resultados de la mejora del bienestar

\section{Conclusiones}

Finalmente, se pueden extraer las siguientes conclusiones en función de los objetivos expuestos para trabajar el bienestar con el grupo de mujeres:

Promocionar y activar la experiencia de salud, entendida como bienestar y empoderamiento a través de la herramienta artística de la acuarela y sus técnicas al agua.

La intervención fue una experiencia que permitió a un grupo de mujeres jóvenes y madres de etnia gitana generar espacios mediante la herramienta artística de la acuarela y sus derivados del agua. Este encuentro reconoció a las mujeres en un nuevo espacio de desarrollo personal y grupal artístico. La muestra colectiva que se realizó en el Centro Juvenil El Sitio de mi Recreo fue el reflejo de la vivencia artística, fortaleciendo las relaciones sociales y vínculos entre ellas. El encuentro semanal en Servicios Sociales de Fuentidueña significó un disfrute y ocio con sus pares y un momento de desconexión de los quehaceres del hogar. Estas conclusiones se enriquecen con lo que nos afirma Arbex:

El apoyo social y la colaboración entre las mujeres puede servir de gran ayuda para muchas. Es importante en este sentido fomentar las relaciones y los vínculos sociales entre ellas, impulsar la comunicación y los encuentros de mujeres gitanas el asociacionismo y la participación social. (Arbex, 2000, p.24) 
LARRAÍN, CORTÉS \& ÁVILA / Criar en colores, nuevas experiencias: una intervención de agua y color con madres jóvenes de etnia gitana en Servicios Sociales de Fuentidueña, Villa de Vallecas

Generar un espacio de intervención, aprendizaje y encuentro con la acuarela para el bienestar y disfrute en las usuarias.

La intervención semanal con las mujeres en Villa de Vallecas fortaleció las relaciones sociales y los lazos comunitarios. Como plantea Belver:

El papel del arte y la creación artística en este tipo de vínculos es sin duda un hecho, el arte y los artistas pueden contribuir, dentro de sus posibilidades, a hacer más llevaderas las condiciones de vida de muchas personas, proporcionándoles medios para sentirse mejor consigo mismo, aislarse de ambientes poco agradables, adquirir autoconfianza, sentirse un poco más felices. (Belver, 2011, p.12)

Evaluar el impacto percibido en la participación de los talleres mediante las escalas de Bienestar Mental WEMWBS (C), la medida paraguas de emociones positivas y negativas y los espacios de reflexión.

Como bien se ha explicado en el apartado anterior, el cuestionario de Bienestar Mental WEMWBS@ entregado al inicio y al final del programa de intervención permitió recoger evidencias sobre cómo había aumentado el bienestar de las mujeres. Esta herramienta, junto con otras evidencias descritas a lo largo de este artículo, ponen de manifiesto las potencialidades de este tipo de intervenciones en las que el arte y el lenguaje plástico pueden ser una herramienta al servicio del bienestar.

\section{Referencias}

Arbex, C. (Instituto de la mujer y Asociación Secretariado General Gitano). (2000). Guía de salud para mujeres gitanas. Madrid, España. Recuperado de https://www. gitanos.org/upload/54/37/20120607133826384.pdf

Ávila, N., Orellana, A., Cano, G.M., Antúnez, N., Claver, L. (2014). Arte, salud y prevención: primeras colaboraciones. Gaceta Sanitaria, 28(6), 501-504. doi: 10.1016/j.gaceta.2014.07.002. Recuperado de: http://www.gacetasanitaria.org/es/ arte-salud-prevencion-primeras colaboraciones/articulo/S0213911114001988/

Ávila, N., Orellana, A., Claver, L., Segura, J., Martínez, M., Borrego, O. (2016). Proyectos comunitarios: propuestas desde la promoción de la salud y la creación artística. Comunidad, 2(18),3. Recuperado de http://comunidadsemfyc.es/ proyectos/

Belver, M.H. (2011). El Arte y la Educación artística en contextos de salud. Arte, Individuo y Sociedad, 23 ( $\mathrm{n}^{\mathrm{o}}$ esp), 11-17.

Cortés, C. Rompen en Femenino. Gitaneidad en Femenino. Mujeres en red. El periódico feminista. Recuperado de http://www.mujeresenred.net/spip. php?article567 
De La Cal, L. (2016). Lita la pintora española más cotizada es gitana, El Mundo. Recuperado de http://www.elmundo.es/cronica/2016/04/16/5707e8e3268e3e0629 8b4677.html.

OMS. (1986). Carta de Ottawa para la Promoción de la Salud. Otawa, Canada: Organización Mundial de la Salud. Recuperado de https://www.paho.org/hq/ dmdocuments/2013/Carta-de-ottawa-para-la-apromocion-de-la-salud-1986-SP.pdf

Ramos-Morcillo A.J, et al. (2015). Expectativas y experiencias de uso de las mujeres gitanas mayores ante los servicios sanitarios de atención primaria. Atención Primaria, 47(4), 213-219. Recuperado de http://www.elsevier.es/es-revistaatencion-primaria-27-pdf-S0212656714002285-S300

Saludy ComunidadGitana,Análisisdepropuestas paralaactuación.(2005). Ministerio de sanidad y consumo: Fundación Secretariado Gitano. Recuperado de https:// www.gitanos.org/centro_documentacion/publicaciones/fichas/18183.html.es

Stewart-Brown, S. \& Janmohamed, K. (2008). Warwick-Edinburgh Mental Wellbeing Scale (WEMWBS) (C) Warwick Medical School. University of Warwick. Recuperado de https://warwick.ac.uk/fac/sci/med/research/platform/wemwbs

Thomson, L. J., Chatterjee, H. J. (2015). Measuring the impact of museum activities on well-being: developing the Museum Well-being Measures Toolkit, Museum Management and Curatorchip, 30(1), 44-62, doi: 10.1080/09647775.2015.1008390.

Thomson, L. J., Chatterjee, H. J. (2013). UCL Museum Wellbeing Measures Toolkit: Positive and Negative Wellbeing Umbrella - Generic C) UCL Museums 2013. Recuperado de https://www.ucl.ac.uk/culture/sites/culture/files/ucl_museum_ wellbeing_measures_toolkit_sept2013.pdf

Thomson, L. J., Chatterjee, H. J. (2014). Assesing well-being outcomes for arts and heritage activities: Development of a Museum Well-being Measures toolkit. Journal of Applied Arts \& Health, 5 (1) 29-50. doi: 10.1386/jaah.5.1.29_1.

Web oficial de Bibliopro https://www.bibliopro.org/que-es/index.html

Web oficial de Madrid Salud http://madridsalud.es

Web oficial de OMS http://www.who.int/es

Agradecimientos

Linda J. Thomson, Senior Research Associate UCL Division of Biosciences

University College London.

Diana Sojo Guttero, Jefa de Sección Centro Madrid Salud, Villa de Vallecas

Educadores sociales de familia: Francisco Miñana López y Mercedes Alonso López. 